\title{
Metabolomic Profiling of Lipids for Biomarker Discovery
}

\section{Hua Dong*}

Department of Entomology and Comprehensive Cancer Center, University of California, Davis, CA 95615, USA

Biomarkers are key molecular or cellular substances that are evaluated as indicators of a biological state. They play an important role in understanding the biological processes, pathogenic processes, and pharmacologic responses. Some of the methods to discover biomarkers in different disease models include enzyme-linked immunosorbent assays (ELISA) [1], biosensors [2,3], gas chromatography/mass spectrometry (GC/MS) [4], liquid chromatography (LC) /MS [5], and LC/MS/MS [6,7]. LC/MS/MS is currently the most powerful tool for metabolomic profiling of biomarker discovery due to its specificity and high sensitivity. Metabolomics is the systematic study of the unique chemical fingerprints of small molecules or metabolite profiles that are related to a variety of cellular metabolic processes in a cell, organ, or organism. Metabolic profiling provides direct and indirect physiologic insights that can potentially be detectable in a wide range of biospecimens. Metabolomic platforms for lipids are important because these signaling molecules regulate many fundamental biological processes. For example, the lipids of the arachidonate cascade, which includes eicosanoids, are the targets of $75 \%$ of the world's pharmaceuticals. This article is focused on the application of metabolomics profiling of lipids and the analytical method for lipids profiling is described elsewhere [8].

Eicosanoids are lipids produced from arachidonic acid including prostaglandins (PGs), epoxyeicosatrienoic acids (EpETrEs), hydroxyeicosatetranoic acids (HETEs), thromboxanes (TXs), leukotrienes (LTs), and lipoxins (LXs). Among these compounds, EpETrEs are of biological importantance from several aspects. EpETrEs are anti-inflammatory, analgesic, cardiovascular protective, and anti-hypertensive $[9,10]$. EpETrEs can be converted to their less biologically active corresponding vicinal diols, dihydroxyeicosatrienoic acids (DiHETrE) by a xenobiotic-metabolizing enzyme, soluble epoxide hydrolase $(\mathrm{sEH})$. Inhibition of $\mathrm{sEH}$ by either $\mathrm{sEH}$ gene knockout or using a selective sEH inhibitor (sEHI) can increase the levels of EpETrEs in vivo and leads to decreased inflammation. sEH has proven to be a therapeutic target for acute inflammation, pain, and cardiovascular diseases [11]. Potent sEHIs have been developed for several decades and have emerged as therapeutic drug agent for treating inflammatory diseases. By using metabolomic profiling method, changes of eicosanoid profile could be evaluated and used as valuable biomarkers for assessing the engagement of sEHI in vivo [7]. For example, a significantly decreased EpETrEs/DiHETrEs ratio, which represents an inflammatory state, was found in a murine model of myocardial infarction by lipids metabolomic profiling. However, with the dramatically increased ratios of EpETrEs/DiHETrEs after drug treatment, sEHI was demonstrated to be highly effective in the prevention of progressive cardiac remodeling post myocardial infarction [12]. This indicates that the ratios of EpETrEs/DiHETrEs are closely related with the function of sEHI. Other studies using lipids metabolic profiling also revealed that either the ratios of EpETrEs/ DiHETrEs or the increased EpETrEs is correlated with sEHI treatment and may be used as a potential biomarker to evaluate the engagement of sEHI [13-17].

20-hydroxyeicosatetraenoic acid (20-HETE), one of the metabolites from arachidonic acid produced by cytochrome P45s (CYP450s), is another important compound of eicosanoids. It is a potent vasoconstrictor and plays an important role in cardiovascular function. In a recent study, 20-HETE was found to be an unexpected biomarker in a selective cyclooxygenase (COX)-2 inhibitor, rofecoxib, mediated cardiovascular events [18]. By metabolomic profiling of the representative oxylipins derived from arachidonic acid and linoleic acid mediated by COXs, CYP450s, and lipoxygenases (LOXs) pathways instead of monitoring arachidonic acid generated oxylipins from COX enzymes only, the authors found a 120-fold increase of 20-HETE after oral administration of rofecoxib for 3 months in a murine model. And this dramatic change is correlated with a significant shorter tail bleeding time which is one of the side effects of rofecoxib. Their work suggested that 20-HETE may potentially be used as a biomarker of rofecoxib induced cardiovascular adverse events.

20-HETE has also emerged as a new biomarker and therapeutic target for hypertension. The vascular actions of 20-HETE are known to be prohypertensive. It is a vasoconstrictor in small arteries and also has natriuretic properties [19]. Sodhi et al. provided evidence that 20 HETE generated by increased endothelial CYP4A2 expression lead to increased angiotensin-converting enzyme (ACE) and angiotensindependent hypertension [20]. One recent study by Chabova et al. demonstrated that combining 20-HETE inhibition with sEH inhibition successfully decreased blood pressure and improved kidney function in Ren-2 renin transgenic rats that have angiotensin-dependent hypertension [21]. Using another hypertensive animal model, Zhang et al. recently found that urinary 20-HETE excretion was increased in adrenocorticotrophic hormone (ACTH)-induced hypertensive rats, while inhibition of 20-HETE production by HET0016, a selective inhibitor of 20-HETE, prevented and reversed ACTH-induced hypertension [22]. Taken together, these evidences suggested 20HETE may be used as a biomarker of vascular dysfunction and as a new therapeutic approach for the treatment of hypertension.

In conclusion, metabolomic profiling of lipids has proven to be a promising tool to gain comprehensive understandings of biological processes. It has played increasingly important roles due to its utility in the identification of candidate biomarkers associated to disease status. Its application has ranged from biological fundamental studies to pharmaceutical research and development, and to clinical research. Biomarker discovery by metabolomic profiling of lipids will have a broad application in the improvement of early diagnostics, patient monitoring and for the evaluation of the safety and efficacy of therapeutic strategies.

*Corresponding author: Hua Dong, Department of Entomology and Comprehensive Cancer Center, University of California, Davis, CA 95615 USA, Tel: 530-752-5109; E-mail: hdong@ucdavis.edu

Received August 31, 2012; Accepted August 31, 2012; Published September 04, 2012

Citation: Dong H (2012) Metabolomic Profiling of Lipids for Biomarker Discovery Biochem Anal Biochem 1:e115. doi:10.4172/2161-1009.1000e115

Copyright: @ 2012 Dong $\mathrm{H}$. This is an open-access article distributed under the terms of the Creative Commons Attribution License, which permits unrestricted use, distribution, and reproduction in any medium, provided the original author and source are credited. 


\section{References}

1. Wiest MM, Watkins SM (2007) Biomarker discovery using high-dimensiona lipid analysis. Curr Opin Lipidol 18: 181-186.

2. Dasilva N, Díez P, Matarraz S, González-González M, Paradinas S, et al. (2012) Biomarker discovery by novel sensors based on nanoproteomics approaches. Sensors (Basel) 12: 2284-2308

3. Dong H, Wang S, Galligan JJ, Swain GM (2011) Boron-doped diamond nano/ microelectrodes for biosensing and in vitro measurements. Front Biosci (Scho Ed) 3: 518-540.

4. Newman JW, Hammock BD (2001) Optimized thiol derivatizing reagent for the mass spectral analysis of disubstituted epoxy fatty acids. J Chromatogr A 925: 223-240.

5. Deems R, Buczynski MW, Bowers-Gentry R, Harkewicz R, Dennis EA (2007) Detection and quantitation of eicosanoids via high performance liquid chromatography-electrospray ionization-mass spectrometry. Methods Enzymol 432: $59-82$.

6. Buczynski MW, Dumlao DS, Dennis EA (2009) Thematic Review Series: Proteomics. An integrated omics analysis of eicosanoid biology. J Lipid Res 50: 1015-1038.

7. Yang J, Schmelzer K, Georgi K, Hammock BD (2009) Quantitative profiling method for oxylipin metabolome by liquid chromatography electrospray ionization tandem mass spectrometry. Anal Chem 81: 8085-8093.

8. Yang J, Dong H, Hammock BD (2011) Profiling the regulatory lipids: another systemic way to unveil the biological mystery. Curr Opin Lipidol 22: 197-203.

9. Spector AA, Norris AW (2007) Action of epoxyeicosatrienoic acids on cellular function. Am J Physiol-Cell Phyiol 292: C996-C1012.

10. Michaelis UR, Fleming I (2006) From endothelium-derived hyperpolarizing factor (EDHF) to angiogenesis: Epoxyeicosatrienoic acids (EETs) and cell signaling. Pharmacol Ther 111: 584-595.

11. Imig JD, Hammock BD (2009) Soluble epoxide hydrolase as a therapeutic target for cardiovascular diseases. Nat Rev Drug Discov 8: 794-805.

12. Li N, Liu JY, Timofeyev V, Qiu H, Hwang SH et al. (2009) Beneficial effects of soluble epoxide hydrolase inhibitors in myocardial infarction model: Insight gained using metabolomic approaches. J Mol Cell Cardiol 47: 835-845.

13. Inceoglu B, Jinks SL, Ulu A, Hegedus CM, Georgi K, et al. (2008) Soluble epoxide hydrolase and epoxyeicosatrienoic acids modulate two distinct analgesic pathways. Proc Natl Acad Sci U S A 105:18901-18906.

14. Wang L, Yang J, Guo L, Uyeminami D, Dong H et al. (2012) Use of a soluble epoxide hydrolase inhibitor in smoke-induced chronic obstructive pulmonary disease. Am J Respir Cell Mol Biol 46: 614-622.

15. Zhang W, Liao J, Li H, Dong $\mathrm{H}$, Bai H, et al. (2012) Reduction of inflammatory bowel disease-induced tumor development in IL-10 knockout mice with soluble epoxide hydrolase gene deficiency. Mol Carcinog.

16. Zhang W, Yang AL, Liao J, Li H, Dong H, et al, (2012) Soluble Epoxide Hydrolase Gene Deficiency or Inhibition Attenuates Chronic Active Inflammatory Bowe Disease in IL-10(-/-) Mice. Dig Dis Sci.

17. Charles RL, Burgoyne JR, Mayr M, Weldon SM, Hubner N, et al. (2011) Redox regulation of soluble epoxide hydrolase by 15-deoxy-delta-prostaglandin $\mathrm{J} 2$ controls coronary hypoxic vasodilation. Circ Res 108: 324-334.

18. Liu JY, Li N, Yang J, Li N, Qiu H, et al. (2010) Metabolic profiling of murine plasma reveals an unexpected biomarker in rofecoxib-mediated cardiovascular events. Proc Natl Acad Sci U S A 107: 17017-17022.

19. Roman RJ (2002) P-450 metabolites of arachidonic acid in the control of cardiovascular function. Physiol Rev 82: 131-185.

20. Sodhi K, Wu CC, Cheng J, Gotlinger K, Inoue K, et al. (2010) CYP4A2induced hypertension is 20-hydroxyeicosatetraenoic acid- and angiotensin IIdependent. Hypertension 56: 871-878.

21. Certíková Chábová V, Walkowska $A$, Kompanowska-Jezierska $E$, Sadowsk J, Kujal P, et al. (2010) Combined inhibition of 20-hydroxyeicosatetraenoic acid formation and of epoxyeicosatrienoic acids degradation attenuates hypertension and hypertension-induced end-organ damage in Ren-2 transgenic rats. Clin Sci 118: 617-632

22. Zhang Y, Wu JH, Vickers JJ, Ong SL, Temple SE, et al. (2009) The role of 20-hydroxyeicosatetraenoic acid in adrenocorticotrophic hormone and dexamethasone-induced hypertension. J Hypertens 27:1609-1616. 